\title{
Health and psychosocial issues of individuals with incomplete and resolving spinal cord injuries
}

\author{
K A Gerhart MS, R L Johnson BS, G G Whiteneck PhD \\ Craig Hospital, 3425 South Clarkson, Englewood, Colorado 80110, USA.
}

Longitudinal data and clinical experience indicate that a greater proportion of spinal cord injuries result in incomplete or resolving neurological lesions. Although it has been reported that persons with incomplete injuries enjoy better functional outcomes, routine contacts with these individuals indicate that many experience problems and complications strikingly similar to those with complete spinal cord injuries. Thus, to document the issues and needs of these individuals, data from Colorado's population-based spinal cord injury surveillance program were analyzed. Of 330 persons registered since January 1, 1986, 121 (37\%) were found to be minimally disabled (Frankel class D or E). Review of medical records and follow up documentation for these individuals indicated that although over $75 \%$ were ambulatory and virtually all were physically independent, more than $80 \%$ did report problems in one or more areas: $21 \%$ had orthopedic issues and $17 \%$ faced additional spinal surgery; $16 \%$ reported neurological deterioration or increased spasticity; $25 \%$ had pain problems; and $16 \%$ had bladder difficulties. Other issues included bowel problems, blood pressure abnormalities, skin breakdown, sexual difficulties, depression, and unemployability. Implications for rehabilitation are discussed in the light of these and other findings.

Key words: spinal cord injuries; rehabilitation; follow up.

\section{Introduction}

In the past, the term 'spinal cord injured' most often evoked an image of a severely handicapped, wheelchair-using individual, who faced a lifetime of paralysis and disability. This image, however, is being changed in 2 ways: first, through efforts by the rehabilitation profession to lessen the extent of its clients' handicaps; and second, through advances in safety engineering, medical technology, and emergency care. Perhaps because of the latter, in recent years there has been an increase in the number of individuals who have less disabling spinal cord injuries.

As evidence, the National Spinal Cord Injury Statistical Center in Birmingham, Alabama, reports that between 1974 and 1984 the number of incomplete spinal cord injuries had increased from $38 \%$ to almost $54 \% .{ }^{1}$ Similarly, the Early Notification System, a comprehensive and population-based surveillance program that registers all spinal cord injuries in the state of Colorado, found that in $198954 \%$ of all reported spinal cord injuries were incomplete. ${ }^{2}$

Individuals who sustain incomplete or less severe spinal cord injuries have relatively better outcomes than individuals who sustain complete lesions at the same vertebral levels. Variables such as level of physical independence achieved, length of rehospitalizations, costs of care and follow up, re-employment, and even survival itself, are associated with the extent of neurological preservation and/or recovery. ${ }^{1,3}$ Despite this seeming advantage, however, Colorado's spinal cord injury surveillance and ongoing follow up efforts indicated that people with minimal spinal cord injuries, despite their extensive neurological recovery and high levels of physical independence, still faced medical and psychosocial obstacles.

Consequently, a needs assessment was undertaken to document the issues requir- 
ing intervention, to identify a role for rehabilitation services in the care and recovery of persons with spinal cord injuries that are minor and/or are quickly resolving, and to guide programmatic development.

\section{Methodology}

Since January 1, 1986, Colorado's spinal cord injury surveillance program, the Early Notification System (ENS) has registered and followed all spinal cord injured Coloradans. Inclusion criteria for the registry are:

(a) traumatic spinal cord injury sustained after January 1, 1986, resulting in any documented degree of transient, resolving, or permanent impairment of strength, sensation, bowel, bladder or sexual functioning;

(b) Colorado residency at the time of injury;

(c) survival until admission to the acute care hospital.

Cases are reported to the central registry at the Colorado Department of Health by clinical contact persons who work with hospitalized patients, and by all of Colorado's hospital medical records departments. The latter are required by Colorado Board of Health regulations to make retrospective reports, utilizing select ICD-9 CM codes as the means of identifying eligible cases. ${ }^{4}$ Since the establishment of these dual surveillance systems, the yearly statewide spinal cord injury incidence rate has been calculated at 34 per million, similar to rates reported elsewhere. ${ }^{1,3.5-9}$

Following report of a case, patient medical records are obtained and reviewed to verify that inclusion criteria have been met, and to extract necessary demographic, epidemiologic, medical and outcome data. Patients are contacted personally to collect additional data, and so support and follow up can be provided. Re-contacts are made at prescribed intervals to assess outcomes.

At the time the study was conducted, 330 cases had met the eligibility criteria. Time post injury ranged from less than one year to just under 4 years. The extent of neurological preservation, or incompleteness of the injury, was coded at the time of discharge from the rehabilitation hospital using the Frankel classification system (Table I). ${ }^{10}$ If the patient had not been referred to a rehabilitation hospital, the Frankel classification at the time of discharge from the last acute care hospital at which he or she received post injury care was utilized.

One hundred and twenty-one people $-37 \%$ of the entire sample-were found to have had minimally disabling injuries: all had been coded as either Frankel class D (functional motor recovery) or Frankel class $\mathrm{E}$ (full recovery). ${ }^{10}$ Fifty-three percent of the 121 had sustained cervical injuries; the remainder had had thoracic, lumbar or sacral insults. Over $75 \%$ of the 121 individuals were ambulatory without orthoses or devices, and virtually all were physically independent.

Approximately two thirds of these 121 individuals had at least some rehabilitation, while one third of the group was discharged directly home from the acute care hospital. The median length of initial acute care hospitalization was 18 days (mean $=24$ days; range 1-81 days), while for those who had rehabilitation, the median length of

Table I Neurological preservation following spinal cord injury ${ }^{10}$ based on Colorado's comprehensive surveillance 1986-1989

\begin{tabular}{lrr}
\hline Level of injury & Number & Percent \\
\hline Frankel class A (no neurological preservation below level of injury) & 126 & 38 \\
Frankel class B (sensory sparing only) & 30 & 9 \\
Frankel class C (motor sparing which is not functional) & 48 & 15 \\
Frankel class D (functional motor sparing) & 6 & 35 \\
Frankel class E (complete recovery) & 5 & 2 \\
Unknown & 330 & 1 \\
Total & & -
\end{tabular}


inpatient stay was 39 days (mean $=49$ days, range 6-136 days). Those in the same database with neurologically complete injuries had a median acute hospital stay of 29 days (mean $=43$ ) and a median inpatient rehabilitation hospitalization of about 82 days (mean $=90$ days).

Identification of the ongoing medical concerns, psychosocial issues, and other problems of those with minimal spinal cord injuries was accomplished in 3 ways:

1 Available medical records were obtained and reviewed. For more than one half of the entire sample, the full rehabilitation record was reviewed. For the remainder-approximately $20 \%$ of those who had rehabilitation, and all of those who were discharged directly home from the acute care hospital-significant portions of the medical record were obtained and reviewed, including physician admission and discharge summaries.

2 For all patients, documentation of follow up contacts made by the Early Notification System itself was reviewed and post discharge health and psychosocial issues were recorded.

3 All patients who could be located $(90 \%$ of the sample), regardless of where or whether they had rehabilitation, were re-interviewed annually. Data relating to medical and health issues and financial, psychosocial and other outcomes were collected.

Broad topic categories of issues, including pain, orthopedic and neurological issues, bladder, bowel and other medical problems, and psychosocial issues, were identified. Specific concerns within each category are presented using descriptive analyses, and specific case descriptions and possible implications are discussed.

\section{Findings}

In total, over $80 \%$ of this sample reported some problem or unmet need. Table II depicts the frequency of these problems.

Regardless of whether or not they had rehabilitation, 25 people $(21 \%)$ were discharged home before there was full orthopedic stability of the fracture site. Ten were still in halo immobilization devices and 12 still wore thoraco-lumbar orthoses. Because of the restrictions imposed by these devices, as well as physician-recommended activity limitations, many of these people needed help at home for bowel care and catheterization, as well as to dress, get up and down stairs, and drive. However, many reported that because they could not get or afford such help, they had not been com-

Table II Areas of problems and needs reported by persons with incomplete spinal cord injuries, Colorado 1986-1989

\begin{tabular}{lcc}
\hline Problem Category & Number & $\begin{array}{c}\text { Percent of } \\
\text { sample }\end{array}$ \\
\hline Orthopedic issues & & \\
$\quad$ Unstable fracture & 25 & 21 \\
$\quad$ Additional surgery & 21 & 17 \\
Neurological issues & 11 & 9 \\
$\quad$ Deterioration & 7 & 6 \\
$\quad$ Spasticity & 12 & 10 \\
$\quad$ Head injury & 2 & 2 \\
$\quad$ Seizures & & 16 \\
Pain & 20 & 17 \\
Orinary system & 21 & 24 \\
Psychosodical & & 10 \\
$\quad$ Finances issues & 29 & 7 \\
$\quad$ Depression & 12 & 9 \\
$\quad$ Olcohol concerns & 9 & \\
\hline
\end{tabular}


pliant with their physicians' orthopedic restrictions.

Seventeen percent of the sample needed additional spinal surgery after completing the initial hospitalization. This included 4 individuals who had had late decompression procedures, 3 others for whom the same was recommended, and 5 who had Harrington rods removed. In addition, 4 individuals underwent-and several others were considering-late fusions due to inadequate bony healing. One person required a full spinal fusion. Even though fully innervated and with weakness only in his hands, a premorbid but non problematic scoliosis got rapidly worse following onset of the spinal cord injury and removal of the halo vest. Immediately prior to fusion, 9 months post spinal cord injury, his spinal curves had progressed to 33, 73 and 27 degrees.

Neurologically, 11 people $(9 \%)$ reported deterioration in strength, sensation, or both. Seven more reported increasing spasticity. Almost $10 \%$ had specific chart references to unequivocal or suspected closed head injury as well. Two other individuals with no documentation of head injury developed seizure disorders in the months following their traumatic spinal cord injuries. For one, the first seizure occurred while driving an automobile. The individual lost control, and the ensuing head-on collision resulted in the death of the occupant of another vehicle.

Thirty people, or $25 \%$ of the sample, reported pain issues. Complaints included neck or back pain, dysesthesias, and joint pain. Most pain was treated conservatively using rest and medication. One individual required admission to a chronic pain rehabilitation program. Among those reporting joint pain, the most dramatic case involved an ambulatory and fully independent 26 year old male who, within 2 years of his cervical central cord injury, presented with avascular necrosis of his hip. Severe pain forced him to curtail his ambulation and activity level. A total hip replacement was done by three years post injury, and the physician indicated that repeat replacements probably will be needed every 10 to 15 years.

Among the over $16 \%$ reporting urinary problems, there were 12 with urinary tract infections requiring treatment, 6 with dribbling, frequency or urgency, and 2 with documented ureteral reflux. Stones, sphincter dyssynergy, and high post void residual urine volumes also were problems, as were decreased bladder capacities.

Other medical problems included 8 reports of bowel problems, 3 of gastric problems, 4 complaints of hypertension and 2 of problematic orthostatic hypotension. In addition, 2 individuals suffered skin breakdown caused by ill-fitting trunk and ankle orthoses, and 2 more reported significant burns. In both of the latter, the heat of an object placed on the lap had been underestimated.

Psychosocial issues covered a wide range of concerns. Of those over 18 years old, $38 \%$ were unemployed at one year following injury. This is over 7 times greater than in Colorado as a whole, which reported an adult unemployment rate of only $5.1 \% .^{11}$ Twenty-four percent of the entire group reported financial difficulties, including 21 individuals who had no way to pay their medical bills or could not otherwise support themselves. Feelings of depression were documented in 12 cases, or $10 \%$ of the sample. Four people were involved in formal counseling, and it was recommended for the remaining 8 but never became a reality because of cost or unavailability of professional services in their areas of the state.

For 6 people with pre morbid histories of alcohol abuse, there was documented concern that the injury would cause a relapse. One other individual reported increased alcohol use by the spouse, and for 2 more, concerns about the injury, finances and litigation had caused their own drinking to increase.

Seven medical records documented sexual difficulties. These included dissatisfaction, loss of function that initially had been present following the injury, and the need for education or training. Finally, 2 individuals reported that their children developed behavioral problems following the parent's injury, and 2 more reported that profound self consciousness kept them from leaving their homes and socializing. 


\section{Discussion}

With more than $80 \%$ of individuals with minimal spinal cord injuries reporting problems or concerns in at least one area, it is clear that good neurological recovery alone does not ensure that there will be no post discharge problems. In fact, the problems reported by individuals with minimal spinal cord injuries are strikingly similar to those of their much more severely disabled counterparts.

Nonetheless, over one third of these individuals never interacted with rehabilitation providers. Among those who were admitted to rehabilitation programs, average lengths of stay were consistently shorter than for patients with complete spinal cord injuries. Thus, because of their frequently shortened acute hospital stays, and their equally short, or non existent rehabilitation admissions, the potential of rehabilitation as a source of follow up support through the adjustment process, and identification of post discharge options may not have been realized. Concern is doubled by the fact that the minimal impairments and disabilities of the study group make it likely that relatively little therapeutic, financial, and vocational intervention would be needed to ensure such positive outcomes as successful adjustment, restoration of self esteem, and resumption of previous family, social, and vocational roles.

Moreover, such patients may be unwittingly set up for failure. Although technically 'independent' when discharged from the hospital, they may still be using spinal immobilization devices or may be under physician instructions to restrict bending, twisting, and other activities. Though clearly not needing further inpatient hospitalization, they may, nonetheless, have difficulty getting up and down stairs safely, dressing, or performing vital hygiene tasks. If lacking sufficient mobility to drive safely, they may be housebound. When these issues are unanticipated, patients face frustration and depression. They may require attendant care for which they have not been prepared. When such care is unavailable, unaffordable or unacceptable, patients may be non compliant, and may risk further injury or com- promise delicate surgeries. This may be an even greater concern than for patients with more severe disabilities whose paralysis may itself limit independence and dictate the need for assistance. Thus, if help in the home is not available, or if funding precludes temporary use of personal care attendants, short term hospitalization at intermediate care centers, or other discharge plans may need to be considered.

The limitations of this study are those inherent in other studies relying on medical records review. Problems the patient failed to report, or the physician chose not to document are not recorded. At the same time, such a methodology may favor those who experience many problems and so are heavy users of follow up care. In addition, the number or severity of long term problems was not correlated with measures of neurological recovery that were more specific than the Frankel classification system, or with the length of acute care or rehabilitation hospital stays. Nonetheless, preliminary data and specific examples illustrate that at least some issues - particularly those relating to coping, adjustment, employment, family concerns, and self esteemmay not be bound to the severity of neurological damage.

In any case, it is clear that this group of individuals is in need of support. Not only is more research needed, but a greater effort to reach these patients programmatically and clinically is required. In that the system of care for those with severe spinal cord injuries is already in place, and the expertise already exists, the next step-the development of programs for patients who may not require extensive inpatient care - is not a large one. Physician education, particularly for those providing primary acute care for individuals with new spinal cord injuries, will be needed. Improved spinal cord injury surveillance, now being conducted in many states, will make identification easier and could be used to help facilitate referral to rehabilitation based programs which too frequently are overlooked for patients with minimal spinal cord injuries. A model might be developed to offer such services as therapy, counseling, re-entry, advocacy and follow up on an outpatient basis. Alterna- 
tive and innovative sources of funding will need to be explored and developed. Clearly, there is a role for rehabilitation in treating the individual with the seemingly resolving but certainly not insignificant spinal cord injury.

\section{Acknowledgements}

This research utilizes data from the Colorado Spinal Cord Injury Early Notification System, a comprehensive collaborative surveillance pro- gram supported by a United States government National Institute on Disability and Rehabilitation Research grant (No. G008535132), awarded to Craig Hospital, and by a United States Centers for Disease Control Cooperative Agreement for the Prevention of Disabilities (No. U59/CCU803364), awarded to the Colorado Department of Health, divisions of Epidemiology and Prevention Programs. Special thanks to George N Richardson MRC and Richard Hoffman MD, MPH for the support and guidance they provide the Early Notification System.

\section{References}

1 Stover SL, Fine PR (1986) Spinal Cord Injury: Facts and Figures University of Alabama, Birmingham, Ab.

2 Colorado Department of Health, Division of Prevention Programs (1990) 1989 Annual Report of the Spinal Cord Injury Early Notification System. Colorado Department of Health, 4210 East llth Ave, Denver, Colorado.

3 Young JS, Burns PE, Bowen AM, McCutchen R (1982) Spinal Cord Injury Statistics. Good Samaritan Medical Center, Phoenix, Az.

4 World Health Organization (1978) International Classification of Diseases. Clinical Modification (ICD9-CM). 9th revision. Edwards Brothers Inc, Ann Arbor, Mi.

5 Fine PR, Kuhlemeier KV, DeVivo MJ, Stover SL (1979) Spinal cord injury: an epidemiological perspective. Paraplegia 17: 237-250.

6 Kraus JF (1980) Injury to the head and spinal cord: the epidemiologic relevance of the medical literature published from 1960-1978. J Neurosurg 53: 3-10.

7 Kraus JF, Franti CE, Riggins RS, Richards D, Borhani NO (1975) Incidence of traumatic spinal cord injury lesions. J Chron Dis 28: 471-492.

8 Kurtzke JF (1975) Epidemiology of spinal cord injury. Exp Neurol 3(2): 163-236.

9 Griffin MR, Opitz JL, Kurland LT, Ebersold MJ, O'Fallon WM (1985) Traumatic spinal cord injury in Olmsted County, Minnesota. Am J Epidemiol 121(6): 884-895.

10 Frankel HL, Hancock DO, Hyslop G, Melzak LS, Michaelis GH, Ungar JDS et al (1969) The value of postural reduction in the initial management of closed injuries of the spine with paraplegia and tetraplegia. Paraplegia 7: 179-192.

11 Colorado Department of Labor and Employment. Personal communication: November 1989 data. 